\title{
Searching for gamma-ray emission from the white dwarf pulsar system AR Sco using Pass 8 Fermi-LAT data
}

\author{
Q. Kaplan ${ }^{1 *}$, P.J. Meintjes ${ }^{1}$, K.K. Singh ${ }^{1,2}$, H.J. van Heerden ${ }^{1}$ \\ 1.) Department of Physics, University of the Free State, PO Box 339, Bloemfontein, 9300 \\ 2.) Astrophysical Sciences Division, Bhabha Atomic Research Centre, Mumbai, India, 400085 \\ E-mail: kaplanq@ufs.ac.za
}

\begin{abstract}
AR Sco is a close compact binary system that shows non-thermal, pulsed and highly linear polarized emission. In this study, a search for high energy gamma-ray emission was conducted between $100 \mathrm{MeV}$ - $500 \mathrm{GeV}$ by analysing the newly available Fermi-LAT Pass 8 data with the newly available Fermi 1.0.1 Science Tools. Binned likelihood analysis was performed using power law, broken power law and log parabola models. From the selected Region of Interest (ROI) centred on AR Sco's position, we calculated a statistical significance of $\sqrt{T S} \leq 4.31 \sigma$ for the integrated gamma-ray activity between $100 \mathrm{MeV}-500 \mathrm{GeV}$ at a photon flux level of $(4.39 \pm 3.24) \times 10^{-9} \mathrm{ph} \mathrm{cm}^{-2} \mathrm{~s}^{-1}$ using the broken power law model. This resulted in $2 \sigma$ photon flux upper-limits from the position of AR Sco. Unbinned likelihood analysis was also done to maximize the probability of detection from this faint gamma-ray emitting region, resulting in a statistical $\sqrt{T S} \leq 3.69 \sigma$ value using a broken power law model with an associated photon flux level of $(3.57 \pm 1.10) \times 10^{-9} \mathrm{ph} \mathrm{cm}^{-2} \mathrm{~s}^{-1}$.
\end{abstract}

7th Annual Conference on High Energy Astrophysics in Southern Africa

28 - 30 August 2019

Swakopmund, Namibia

${ }^{*}$ Speaker. 


\section{Introduction}

The close binary system AR Scorpii (henceforth AR Sco) [1] features non-thermal dominated multi-frequency emission from radio to X-rays. This source has been the focus of intensive multiwavelength observational and theoretical investigation since the discovery of pulsed optical and radio emission by Marsh et al. in 2016 [1]. This binary system consists of a $0.81-1.29 M_{\odot}$ highly magnetic white dwarf (WD) with a surface magnetic field B $\leq 500 \mathrm{MG}$ [2, 3] orbiting a 0.28-0.45 $M_{\odot}$ M5 spectral-type dwarf star with an orbital period of $P_{\text {orb }}=3.56$ hours. The WD in the system also exhibits variable pulsar-like emission at the rotational period of $P_{\text {spin }}=117 \mathrm{~s}$, with strong supplementary pulsations at $118 \mathrm{~s}$ which is the beat period between the spin an orbital period [2].

Optical observations utilizing the South African Astronomical Observatory (SAAO) HIPPO Polarimeter [4] on the $1.9 \mathrm{~m}$ telescope reveals strong linear polarization at levels up to $40 \%$ with significantly lower levels of circular polarization $(<10 \%)[2,3]$. The morphology and pulse profile of the linear polarization are similar to that of the Crab pulsar, which is a young isolated neutron star pulsar that emits radiation from radio to $\mathrm{TeV}$ energies, e.g. [5]. The nature of the linear polarization profile is also consistent with synchrotron emission produced in the magnetic field of a rotating magnetic dipole, strengthening the notion that the WD in AR Sco may in fact be a "WD pulsar" which can produce non-thermal pulsar-like emission. Also, the possibility that nearly the entire Spectral Energy Distribution (SED) may be dominated by non-thermal emission sparked enormous interest among theorists, especially how non-thermal emission is being produced $[6,7]$.

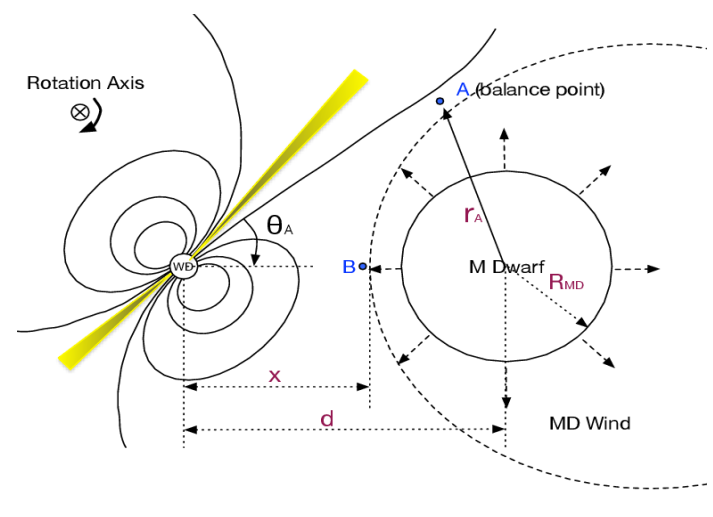

(a)

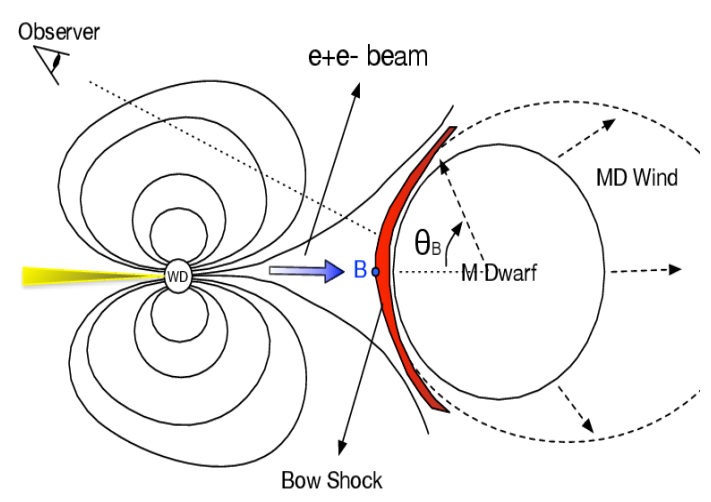

(b)

Figure 1: Proposed schematic illustration of the WD/M-dwarf binary in AR Sco, where (a) shows the increase of emission from the WD as the electron/positron beam approaches the M-dwarf wind (point A), and (b) shows the episode when the open field lines of the WD interacts with the Mdwarf to form a bow shock, where electrons are accelerated to relativistic energies. Adopted from Geng et al. [6]

Geng et al. [6] modelled the emission as being the result of the interaction between the highly magnetized WD and the M5-type secondary dwarf star (see Fig. 1). In this model it is proposed that the WD is a nearly perpendicular rotator where both magnetic poles sweep across the Mtype star periodically. The interaction between the particle beam streaming out from the open field line regions and the M-type wind would lead to the formation of a bow shock, where the 
relativistic particles can be accelerated by e.g. magnetic pumping and shocks to produce possible synchrotron radiation from relativistic electrons in the magnetosphere of the secondary. However, other non-thermal theoretical models has also been developed postulating very high energy gammaray production through a hadronic channel like $\pi^{o}$ is also possible [8], as well as inverse-Compton scattering [3,9]. It is also suggested that the relativistic electrons are trapped within the WD's magnetic field lines due to the magnetic mirror effect and that the pulsed emission originates from the first magnetic mirror point [9]. Both the models presented by Geng et al. 2016 [6] and Takata et al. 2018 [9] suggest non-thermal emission inside the binary system.

Given the current knowledge we have on AR Sco and its non-thermal nature, an attempt is being made to search for continuum gamma-ray emission using a 10-year Fermi-LAT data set with Fermi's updated Pass 8 data analysis framework and background models. If low-level gammaray emission can be observed in Fermi-LAT data, it will definitely spark enormous interest in this source and result in highly magnetized rotating WDs in close binaries being considered as a new class of gamma-ray sources.

\section{Fermi-LAT Analysis}

Pass 8 Release 3 (P8R3) Fermi-LAT data from the past decade (4Aug2008-18Mar2019) were extracted from the Fermi Science Support Center (FSSC) [10] in the energy range of $100 \mathrm{MeV}$ to $500 \mathrm{GeV}$. The Pass 8 data set provides a better determination of the diffuse galactic gammaray emission and a significant improvement in terms of energy resolution from previous FermiLAT pipelines. SOURCE class event files (evclass=128, evtype=3) corresponding to the P 8R3-SOURCE-V6 instrument response function, within a Region of Interest (ROI) of $10^{\circ}$ centred at AR Sco (RA: $16^{h} 21^{m} 47.28^{s}$, Dec: $-22^{\circ} 53^{\prime} 10.39^{\prime \prime}, \mathrm{J} 2000$ ), were considered during analyses. Standard binned and un-binned likelihood analyses were performed using the recently released Fermi Tools software, Fermi 1.0.1. All the point sources in the fourth Fermi-LAT catalogue (4FGL) located within the ROI, along with recent Galactic diffuse and extragalactic isotropic background emission models ${ }^{1}$, were used to generate a background model for subtraction, keeping their spectral shapes the same as defined in the 4FGL catalog. As AR Sco is not listed in the 4FGL catalogue, the methodology by manually adding the source to the model centred at its coordinates was used, parameterizing it with a power law spectral shape at first. The associated parameters of spectral models of all point sources within a $1^{\circ}$ radius, including the Galactic and isotropic background models, were chosen to be free to vary while performing the likelihood fitting. After the power law model was added, both broken power law and log parabola models were also used to do a likelihood fit. Due to convergence issues, sources for the broken power law model further away than $15^{\circ}$ with insignificant TS has been discarded to allow for a better overall convergence and fit.

\section{Results and Discussion}

The resulting integrated flux above $100 \mathrm{MeV}$ with corresponding model parameters from the different models used during binned and un-binned likelihood analyses are presented in Table 1

\footnotetext{
${ }^{1}$ https://fermi.gsfc.nasa.gov/ssc/data/access/lat/BackgroundModels.html
} 
and 2 respectively. These results of our present binned analysis in the energy range of $100 \mathrm{MeV}$ - $500 \mathrm{GeV}$ reveal low level emission at the $\leq 4.31 \sigma$ significance level from the position of AR Sco using a broken power law model, where the statistical significance is calculated as $\sigma=\sqrt{T S}$, [11]. Using the similar model parameters as with the binned analysis, un-binned likelihood results revealed lower significant results with $\mathrm{a} \leq 3.40 \sigma$ significance level (see Table 2).

Table 1: Spectral model parameters obtained for the region centred on AR Sco in the energy interval $100 \mathrm{MeV}-500 \mathrm{GeV}$ using binned likelihood analysis. Optimization of AR Sco was done using a power law $(\Gamma=-(2.7 \pm 0.8))$, broken power law $\left(\Gamma_{1}=-(1.00 \pm 0.31), \Gamma_{2}=-(3.83 \pm\right.$ $1.81))$ and $\log$ parabola $(\alpha=(1.30 \pm 0.38), \beta=(0.92 \pm 0.32))$ to determine the likelihood of detection of gamma-rays from the ROI centred on AR Sco. Here $N_{\text {pred }}$ refers to number of predicted counts.

\begin{tabular}{|ccccc|}
\hline \multicolumn{5}{|c|}{ Binned Likelihood Analyses } \\
\hline \hline Spectral model & Flux(photons/cm $/$ /s) & $\mathrm{N}_{\text {pred }}$ & TS & Significance \\
\hline Power Law & $5.75 \pm 3.08 \times 10^{-9}$ & 1116.91 & 9.02 & $\leq 3.00 \sigma$ \\
Broken Power Law & $4.39 \pm 3.24 \times 10^{-9}$ & 1109.37 & 18.54 & $\leq 4.31 \sigma$ \\
Log Parabola & $2.86 \pm 2.12 \times 10^{-9}$ & 823.98 & 15.15 & $\leq 3.89 \sigma$ \\
\hline
\end{tabular}

Table 2: Spectral model parameters obtained for the region centred on AR Sco in the energy interval $100 \mathrm{MeV}$ - $500 \mathrm{GeV}$ using un-binned likelihood analysis. Optimization of AR Sco was done using a power law $(\Gamma=-(2.8 \pm 0.29))$, broken power law $\left(\Gamma_{1}=-(1.34 \pm 0.28), \Gamma_{2}=-(4.99 \pm 0.43)\right.$ and $\log$ parabola $(\alpha=(0.03 \pm 0.20), \beta=(1.06 \pm 0.78))$ to determine the likelihood of detection of gamma-rays from the ROI centred on AR Sco. Here $N_{\text {pred }}$ refers to number of predicted counts.

\begin{tabular}{|ccccc|}
\hline \multicolumn{5}{c|}{ Unbinned Likelihood Analyses } \\
\hline \hline Spectral model & Flux $\left(\right.$ photons $\left./ \mathrm{cm}^{2} / \mathrm{s}\right)$ & $\mathrm{N}_{\text {pred }}$ & TS & Significance \\
\hline Power Law & $4.95 \pm 2.82 \times 10^{-9}$ & 967.93 & 6.52 & $\leq 2.55 \sigma$ \\
Broken Power Law & $3.57 \pm 1.10 \times 10^{-9}$ & 941.23 & 13.68 & $\leq 3.69 \sigma$ \\
Log Parabola & $2.70 \pm 1.96 \times 10^{-9}$ & 752.31 & 13.52 & $\leq 3.67 \sigma$ \\
\hline
\end{tabular}

After the likelihood analysis had been performed, a SED was plotted using e.g. the power law model over 9 energy bins (see Fig. 2a). The SED was obtained using the Fermi build-in function bdlikeSED with the binned likelihood results. The energy flux values can be considered as $2 \sigma$ upper limits based on a limiting TS $<4$ value per bin due to the low overall significance of AR Sco. The distribution of TS values per energy bin (see Fig. 2b) also illustrates that the most significant emission is concentrated in the energy bins below $3 \mathrm{GeV}$. Due to Fermi's low spatial resolution at lower energies, it may be possible for other nearby sources to still contribute to the observed emission, resulting in larger significant values.

Residual and TS maps were also created to examine the different emission regions within the ROI and to visualize the emission excess, if any, from the coordinates centred on AR Sco (see 
Fig 3). From this, some level of emission excess centred on the coordinates of AR Sco can be seen. Further investigation, however, still has to be done to verify that the upper-limit emission is observed from AR Sco itself and not from nearby sources within the ROI. Since most of the emission originate from an energy level below $10 \mathrm{GeV}$ (see Fig. 2b) and the fact that AR Sco lies within the Galactic plane, it is difficult to distinguish if the detected gamma-rays come from AR Sco or from nearby sources due to Fermi's low spatial resolution at lower energies. It can be seen from Fig. 3 that a significant amount of residual emission is noticeable to the left of the position centred on AR Sco, which may well be attributed to the contribution from the Galactic plane. This, however, needs to be confirmed in future studies since both Galactic and isotropic background models were included during analysis.

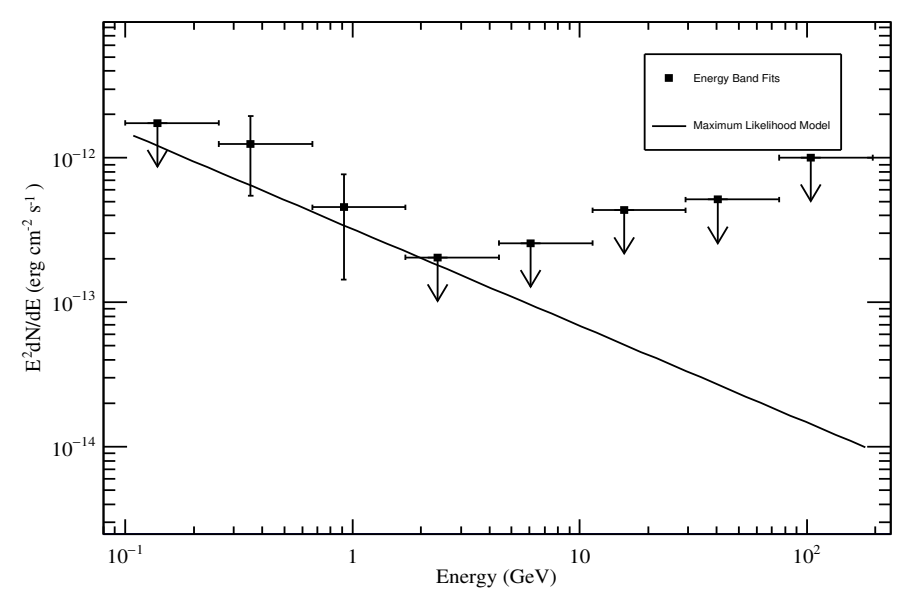

(a) Energy flux SED obtained using bdlikeSED.

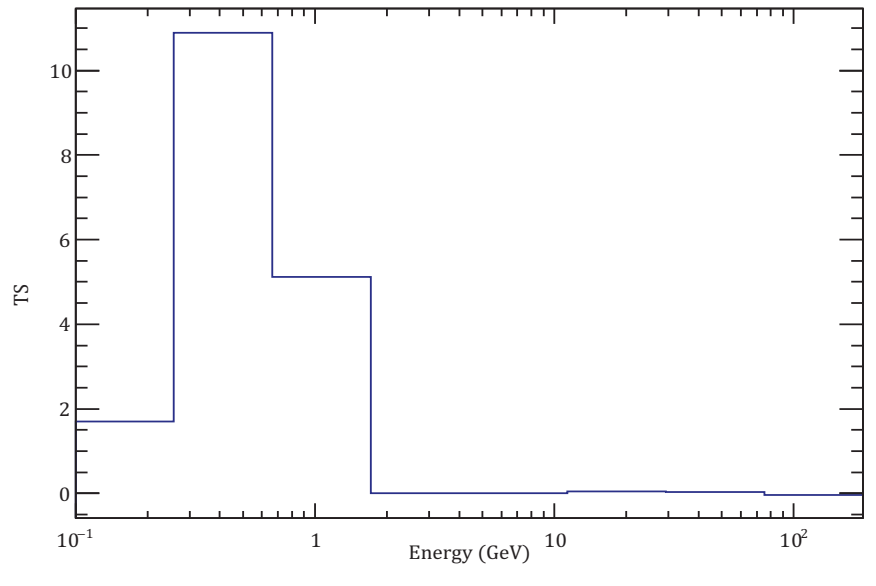

(b) TS values vs energy of $\gamma$-ray photons.

Figure 2: The flux values are $2 \sigma$ upper limits based on a limiting TS $<4$ value per bin. Distribution of high energy $\gamma$-rays are more likely to be detected at lower energies.

\section{Conclusion}

The results from a binned likelihood analysis on a ROI with a radius of $10^{\circ}$ centred on the position of AR Sco, optimized with power law, broken power law and log parabola models, revealed low-level gamma-ray flux between $100 \mathrm{MeV}-500 \mathrm{GeV}$ at the $\leq 4.31 \sigma$ level by considering the broken power law as the best fit. Unbinned likelihood analysis also revealed a similar $\leq 3.69 \sigma$ statistical significance level, albeit at lower levels, which correlates closely with the analyses done using binned likelihood analyses. This, however, is below the $5 \sigma$ Fermi-LAT threshold required for a definite detection. The location of AR Sco inside the Rho Ophiuchi (Rho Oph) molecular cloud complex combined with the poor spatial resolution of Fermi-LAT at lower energies, complicates any positive identification of low-level gamma-ray activity at the location that coincides with the position of AR Sco. With the upcoming Cherenkov Telescope Array (CTA), with a much better sensitivity and resolution than that of Fermi, it may be possible to detect and resolve gamma-ray emission from the region centred in AR Sco. 


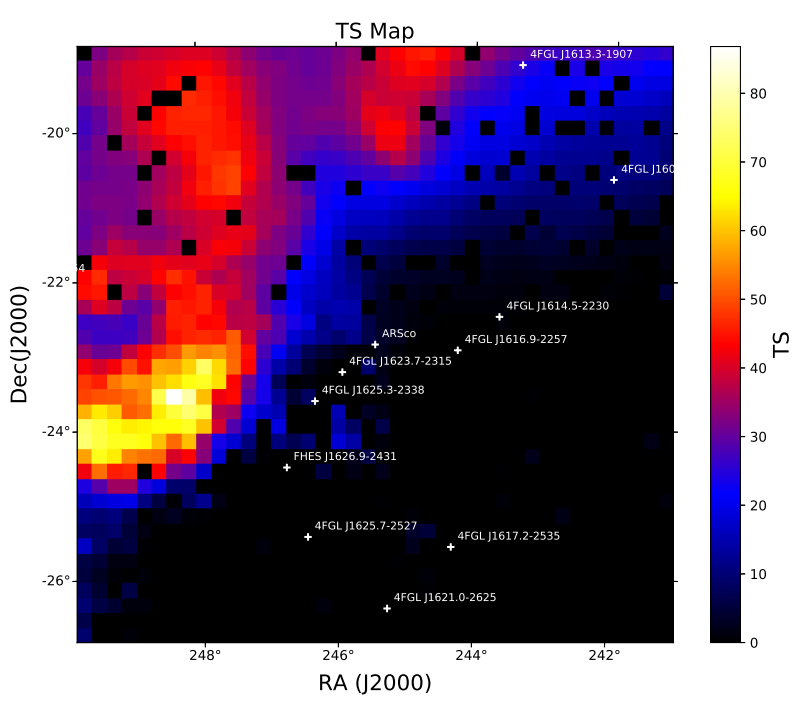

(a) TS map centred on AR Sco

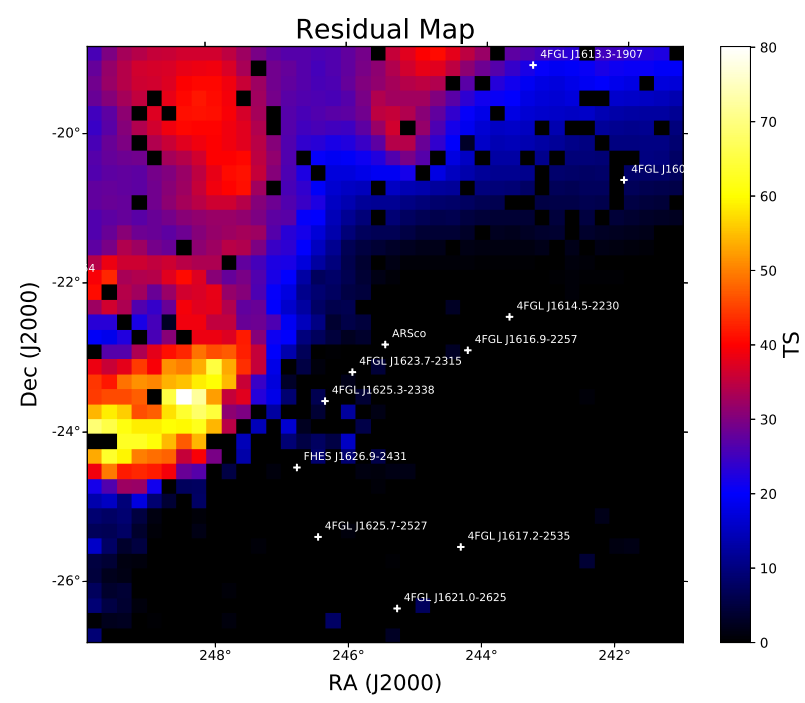

(b) Residual map centred on AR Sco

Figure 3: Plots of AR Sco's TS values within its ROI where (a) shows the TS map centred on AR Sco and (b) shows the residual map centred on AR Sco. If we compare these two maps we can see some excess from the coordinates centred on AR Sco in (a) but not in (b).

\section{Acknowledgements}

The authors thanks the organisers for the invitation as well as the University of the Free State (UFS) Research directorates and SA GAMMA for the financial support. The Fermi-LAT analysis was performed using the UFS High Performance Computing (HPC) Unit.

\section{References}

[1] T. R. Marsh et al., A radio-pulsing white dwarf binary star, Nature 537 (2016) 374

[2] D.A.H. Buckley, P.J. Meintjes, S.B. Potter, T.R. Marsh \& B.T. Gänsicke, Polarimetric evidence of a white dwarf pulsar in the binary system AR Sco, Nature Astronomy 1 (2017) 0029

[3] D.A.H. Buckley, S.B Potter, P.J. Meintjes, T.R. Marsh and B.T. Bänsicke , Polarimetric evidence of the first white dwarf pulsar: The Binary System AR Scorpii, Galaxies 6 (2018) 14

[4] S.B. Potter et al., Polarized QPOs from the INTEGRAL polar IGRJ14536-5522 (= Swift J 1453.4 - 5524), MNRAS 402 (2010) 1161

[5] L. Kuiper et al., The Crab pulsar in the 0.75-30 MeV range as seen by CGRO COMPTEL. A coherent high-energy picture from soft X-rays up to high-energy gamma-rays, $A \& A 372$ (2001) 918

[6] J.-J. Geng, B. Zhang, Y.-F. Huang A model of white dwarf pulsar AR Sco, Astrophys. J. Lett. 831 (2016) L1 
[7] J.I. Katz, AR Sco: Presenting a white dwarf synchronar, Astrophys. J. 835 (2017) 150

[8] W. Bednarek, Hadronic modelfor the non-thermal radiation from the binary system AR Scorpii, MNRAS 476 (2018) L10-L14

[9] J. Takata, C. -P. Hu, L.C.C. Lin, P.H.T. Tam, P.S. Pal, C.Y. Hui, A.K.H Kong and K.S. Cheng, A non-thermal pulsed X-ray emission of AR Scorpii, The Astrophysical Journal 853(2) (2018), 106

[10] J. McErny et al., Fermi Science Support Centre, https://fermi.gsfc.nasa.gov/ssc/ , Date accessed: 18 June 2019

[11] J.R. Mattox, D.L. Bertsch, J. Chiang, The Likelihood Analysis of Egret Data, The Astrophysical Journal 461 (1996), 396-407 